\title{
In vitro secretion of metabolic end-products by piscine haemoflagellates Cryptobia salmositica and C. bullocki (Kinetoplastida: Bodonidae) and the relationship of these products to the $\mathrm{pH}$ in the medium
}

\author{
Bernadette F. Ardelli ${ }^{1,2}$ and Patrick T.K. Woo ${ }^{1}$ \\ ${ }^{1}$ Department of Zoology and Axelrod Institute of Ichthyology, University of Guelph, Guelph, Ontario, N1G 2W1, Canada; \\ ${ }^{2}$ Current address: Institute of Parasitology, McGill University, 21-111 Lakeshore Road, Ste-Anne de Bellevue, Quebec, H9X \\ $3 \mathrm{~V} 9$, Canada
}

Key words: Cryptobia, pyruvate, lactate, hydrogen peroxide, $\mathrm{pH}$

\begin{abstract}
Pathogenic and nonpathogenic strains of Cryptobia salmositica Katz, 1951 and C. bullocki Strout, 1965 produced hydrogen peroxide, pyruvate and lactate under in vitro conditions in Minimum Essential Medium (MEM). As parasite number increased, the phenol red in the medium changed from red to yellow. This change was not associated with a decrease in $\mathrm{pH}$, or an increase in pyruvate or lactate, but was correlated with an increased secretion of hydrogen peroxide. Parasites incubated at $10^{\circ} \mathrm{C}$ in medium at $\mathrm{pH}$ 6.0, 6.5, 7.0 and 7.3 were active for about 1 week with decreasing activity in the absence of serum. Parasites in saline ( $\mathrm{pH} 6.0,6.5,7.0$ and 7.3) were nonmotile within $24 \mathrm{~h}$ and were dead in about 1 week. This suggests that these Cryptobia spp. are sensitive to changes in $\mathrm{pH}$ and require medium which is buffered, either with serum or Hepes.
\end{abstract}

Cryptobia salmositica Katz, 1951 and Cryptobia bullocki Strout, 1965 are members of the Kinetoplastida, with life cycles involving both a piscine host and a leech vector (Woo and Poynton 1995). Parasites of the genus Cryptobia are etiological agents of diseases affecting economically important fish, but they also parasitise many fish without any apparent pathogenicity. Cryptobia spp. that infect fish have structures that are typical of all members of the Kinetoplastida, including a kinetoplast-containing mitochondrium and glycosomes (Ardelli et al. 2000).

None of the Kinetoplastida studied to date are capable of fully degrading glucose to carbon dioxide under aerobic conditions. A great proportion of glucose carbon is excreted into the medium as fermentative metabolites which differ according to the species of parasite (Opperdoes 1995) and may include carboxylic acids. As parasitic protozoans multiply in culture medium, these carboxylic acids accumulate and slowly acidify the medium (Zinsstag et al. 1991). Acidity is often assessed with a $\mathrm{pH}$ indicator, such as phenol red, which changes from red (alkaline) to yellow (acidic). Ardelli and Woo (1998) demonstrated that increasing the concentration of Hepes (from $25 \mathrm{mM}$ to $100 \mathrm{mM}$ ) in MEM yielded significantly larger numbers of $C$. salmositica. They suggested that increasing the amount of buffer allowed the $\mathrm{pH}$ to remain relatively constant (at alkaline $\mathrm{pH}$ ) for a longer period and resulted in a higher yield of parasite. As part of an ongoing study to improve methods of culturing haemoflagellates, our objectives in the present study were to examine (1) some of the metabolic end-products produced by $C$. salmositica (pathogenic and nonpathogenic strains) and C. bullocki under in vitro conditions and (2) the relationship of these products to the $\mathrm{pH}$ of the medium.

\section{MATERIALS AND METHODS}

In vitro culture of Cryptobia spp. strains. A cloned strain of the pathogenic C. salmositica was used to infect rainbow trout Oncorhynchus mykiss (Walbaum). The strain was initially isolated from the leech Piscicola salmositica and details of the cloning of the parasite (Woo 1979) and fish maintenance (Ardelli and Woo 1998) have been described. The pathogenicity of $C$. salmositica is maintained by serial passage in rainbow trout. To culture pathogenic $C$. salmositica, blood was withdrawn from an infected trout and inoculated aseptically into sterile culture flasks containing MEM supplemented with Hank's salts, $100 \mathrm{mg} / \mathrm{ml} \mathrm{L-}$ glutamine, $25 \mathrm{mM}$ Hepes and $25 \%$ heat-inactivated fetal bovine serum (FBS). After three days, an aliquot containing parasites, but no host cells, were transferred to sterile culture flasks containing MEM. The nonpathogenic C. salmositica was attenuated through serial in vitro culture in MEM (Woo and Li 1990) and is infective to trout but does not cause disease (Li and Woo 1995). C. bullocki was initially isolated from the blood of a southern flounder Paralichthys lethostigma (Jordau et Gilbert) by Dr. E. Burreson and has since been maintained in MEM (Woo and Thomas 1991).

Experimental design. MEM was obtained as a powder (Gibco) and a $1.2 \mathrm{~g} / 1$ stock solution was prepared in sterile water. Horseradish peroxidase (HRPO - Type II, salt-free powder; Sigma) was dissolved in $0.05 \mathrm{M}$ potassium phosphate 
buffer ( $\mathrm{pH} 7.0$ ) at a concentration of $5 \mathrm{mg} / \mathrm{ml}$. Aliquots were kept frozen at $-80^{\circ} \mathrm{C}$ until use. Hydrogen peroxide was obtained from Sigma as a $30 \%$ solution and stored at $4{ }^{\circ} \mathrm{C}$.

Eighty tissue culture flasks were divided into eight groups (A1, A2, B1, B2, C1, C2, D1, and D2; n = 10/group). Flasks in Groups A1, B1, C1 and D1 contained $50.0 \mathrm{ml}$ of MEM supplemented with $10 \mathrm{mg} / \mathrm{ml}$ of phenol red (MEM+PR) and flasks in Groups A2, B2, C2 and D2 contained $50.0 \mathrm{ml}$ of MEM, but no phenol red (MEM-PR). Each flask in Groups B1 and B2 was inoculated with 100,000 cells of pathogenic $C$. salmositica, C1 and C2 with 100,000 cells of nonpathogenic C. salmositica and D1 and D2 with 100,000 cells of $C$. bullocki. Groups A1 and A2 were control flasks and did not contain parasites. Flasks were sampled bi-weekly $(2.5 \mathrm{ml})$ and the number of parasites, pyruvate, lactate, hydrogen peroxide and $\mathrm{pH}$ of the culture medium were determined. The number of parasites were counted using a haemocytometer (Archer 1965 ) and the $\mathrm{pH}$ of the medium was determined using a $\mathrm{pH}$ meter.

Pyruvate assay. Pyruvate was determined enzymatically. The method for determining pyruvate depended on the oxidation of reduced nicotinamide-adenine dinucleotide (NAD) in the presence of lactate dehydrogenase at $\mathrm{pH}$ 7.0. A Tris buffer was added to the supernatant to obtain a $\mathrm{pH}$ of 7.0, and the oxidation of reduced NAD was measured spectrophotometrically by the change in extinction at its peak absorption of $340 \mathrm{~nm}$. The concentration of pyruvate in the sample was obtained from a reference scale established with pyruvate solutions at 50, 100 and $200 \mathrm{mmol} / \mathrm{l}$ (Sigma).

To determine pyruvate, $2.0 \mathrm{ml}$ of MEM was pipetted into a centrifuge tube containing $4.0 \mathrm{ml}$ of cold $8 \%$ perchloric acid (to precipitate proteins). Proteins were removed (by centrifugation for $3 \mathrm{~min}$ at $10,600 \mathrm{~g}$ ) and $2.0 \mathrm{ml}$ of supernatant was added to a cuvette containing $0.5 \mathrm{ml}$ of $1.5 \mathrm{M}$ Tris buffer. After mixing, $0.5 \mathrm{ml}$ of $1.0 \mathrm{mg} / \mathrm{ml}$ of NADH was added and the absorbance read at $340 \mathrm{~nm}$ against water as the reference. Lactate dehydrogenase $(0.05 \mathrm{ml} ; 1000 \mathrm{U} / \mathrm{ml})$ was added, and the decrease in absorbance read at $340 \mathrm{~nm}$ (Marbach and Weil 1967).

Lactate assay. Lactate was determined enzymatically. The procedure was based on the principle that lactic acid is converted to pyruvate and hydrogen peroxide by lactate oxidase. In the presence of the hydrogen peroxide formed, peroxidase catalyses the oxidative condensation of chromogen precursors to produce a coloured dye with an absorption maximum at 540 $\mathrm{nm}$. The increase in absorbance at $540 \mathrm{~nm}$ is directly proportional to the lactate concentration in the sample.

To determine lactate, a $1.0 \mathrm{ml}$ aliquot of a solution containing $400 \mathrm{U} / 1$ of lactate oxidase, $2400 \mathrm{U} / 1$ horseradish peroxidase and chromogen precursors in $0.2 \mathrm{M}$ glycine buffer (Sigma) was pipetted into a cuvette. Ten microlitres of MEM were added and allowed to react with the lactate mixture for $10 \mathrm{~min}$. The absorbance was read at $540 \mathrm{~nm}$ using water as the reference (Marbach and Weil 1967).

Hydrogen peroxide assay. The technique used for the measurement of hydrogen peroxide is based on the horseradish peroxidase (HRPO)-dependent conversion of phenol red by hydrogen peroxide $\left(\mathrm{H}_{2} \mathrm{O}_{2}\right)$ into a compound (not yet identified) with an increased absorbance at 600-610 nm. To determine nanomoles of $\mathrm{H}_{2} \mathrm{O}_{2}$ produced per cell, standard curves were prepared using dilutions of a $\mathrm{H}_{2} \mathrm{O}_{2}$ solution of known molarity (Sigma). To $1-\mathrm{ml}$ volumes of MEM, $10 \mu \mathrm{l}$ of $\mathrm{H}_{2} \mathrm{O}_{2}$ were added to result in final concentrations ranging from 1 to $50 \mu \mathrm{M}$. The tubes were incubated at $25^{\circ} \mathrm{C}$, and brought to $\mathrm{pH} 12.5$ by the addition of $10 \mu \mathrm{NaOH}(1 \mathrm{~N})$. The absorbance was read at $600-610 \mathrm{~nm}$ against a blank containing $1 \mathrm{ml}$ of MEM, $10 \mu 1 \mathrm{H}_{2} \mathrm{O}_{2}$ and $10 \mu \mathrm{l} \mathrm{NaOH}(1 \mathrm{~N})$. A wavelength of $610 \mathrm{~nm}$ was chosen, as the $\mathrm{H}_{2} \mathrm{O}_{2}$ concentration in the 1-60 $\mu \mathrm{M}$ range, is linear, and corresponds to 1-60 nmoles of $\mathrm{H}_{2} \mathrm{O}_{2}$ per $\mathrm{ml}$.

To determine hydrogen peroxide, a 1.0-ml aliquot of MEM was added to a cuvette containing horseradish peroxidase (10 $\mu 1$ of a $50 \mu \mathrm{g} / \mathrm{ml}$ solution) and incubated at room temperature for 5 minutes. $\mathrm{NaOH}(10 \mu \mathrm{l}$ of $1 \mathrm{~N})$ was added to the cuvette to adjust the $\mathrm{pH}$ to 12.5 ; this was to eliminate changes in the absorbance of phenol red due to its behaviour as a $\mathrm{pH}$ indicator. The absorbance was read at $610 \mathrm{~nm}$ against a blank containing no horseradish peroxidase. The amount of hydrogen peroxide in the culture medium was extrapolated from the standard curve and expressed in $\mathrm{mM} / 1$ (Pick and Keisari 1980).

Statistical analysis. Data were analysed using a one-way analysis of variance (ANOVA). When the distribution was not normal and the variances were unequal, an ANOVA on ranks was used. Significantly different results were analysed using a multiple comparison procedure (Student-Newman-Keuls test) for equal sample sizes. Significance was evaluated at $P \geq 0.05$.

\section{RESULTS}

Pathogenic and nonpathogenic $C$. salmositica and $C$. bullocki multiplied readily in both MEM+PR medium or MEM-PR medium. The pathogenic C. salmositica did not multiply as readily as nonpathogenic C. salmositica or $C$. bullocki in either medium. In MEM+PR medium a significant difference $(p=0.0830)$ in number was not detected between $C$. salmositica (nonpathogenic) and C. bullocki. However in MEM-PR medium $C$. bullocki multiplied faster than nonpathogenic $C$. salmositica and this was significantly different at $5(p=0.0309), 7(p=0.0408)$ and $11(p=0.0142)$ weeks post inoculation (p.i.). Parasite numbers peaked at 7 weeks p.i. and then declined (in either MEM+PR or MEM-PR) (Fig. 1).

The phenol red in the culture medium changed from red to yellow as parasite number increased. However, the $\mathrm{pH}$ remained constant at 7.4 until 9 weeks after incubation at which time it decreased to 7.2. This observation was the same for both strains of C. salmositica and for C. bullocki. Also, the $\mathrm{pH}$ of the MEM-PR medium did not change as parasite number increased.

To determine the effects of $\mathrm{pH}$ on the viability of Cryptobia spp., parasites were incubated in MEM (with or without serum supplement) or saline at $\mathrm{pH} 6.0,6.5$, 7.0 and 7.3. Parasites in MEM without serum were active and motile for $24 \mathrm{~h}$, but became sluggish after 1 week. However parasites in medium containing serum were still active and motile after 1 week while those in saline were dead by 24 hours. 


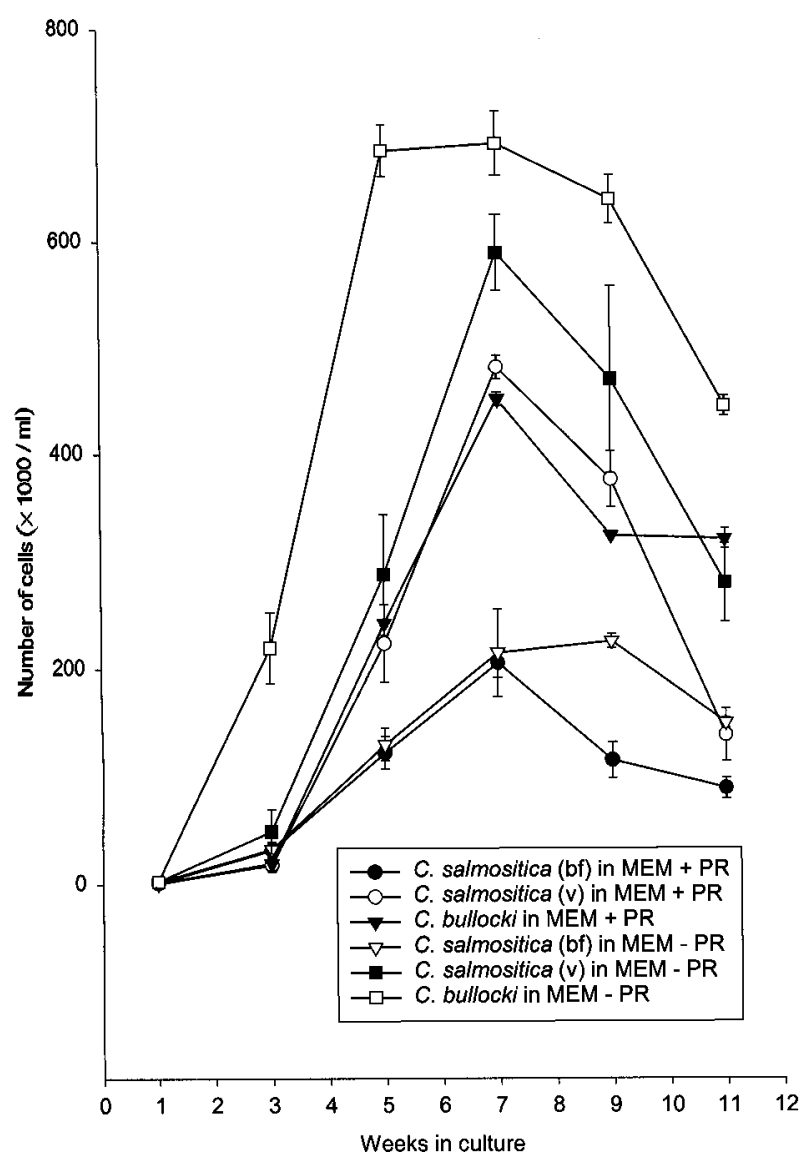

Fig. 1. Multiplication of Cryptobia salmositica (pathogenic and nonpathogenic strains) and Cryptobia bullocki in Minimum Essential Medium with (MEM+PR) or without (MEMPR) phenol red. bf - pathogenic C. salmositica; $\mathrm{v}$ - nonpathogenic C. salmositica.

Hydrogen peroxide was not detected in MEM-PR medium and in control flasks without parasites. It was only detected in flasks containing parasites and phenol red (Table 1). Levels of hydrogen peroxide were low at 1-4 weeks in pathogenic $C$. salmositica but increased at 9 weeks p.i. The nonpathogenic $C$. salmositica produced significantly $(p=0.0205)$ higher amounts of hydrogen peroxide than the pathogenic $C$. salmositica or $C$. bullocki 11 ( $p=0.0487)$ weeks p.i. In general, hydrogen peroxide levels increased throughout the study and were highest at 11 weeks p.i. (Table 1).

In MEM+PR medium pyruvate was first detectable at 1 week p.i. of parasites. Generally, its levels peaked at 7 (for nonpathogenic C. salmositica and C. bullocki) or 9 (pathogenic C. salmositica) weeks p.i., and then declined. Pathogenic $C$. salmositica produced lower amounts of pyruvate than either the nonpathogenic $C$. salmositica or $C$. bullocki at 3-11 weeks p.i. In contrast, C. bullocki produced higher amounts of pyruvate than either strain of $C$. salmositica between 3 and 7 weeks p.i. (Table 2). Similar results were obtained with MEMPR medium (Table 2).

In MEM+PR medium lactate was detectable at 1 week p.i. Lactate levels fluctuated over the course of the study, and peaked at 3 (pathogenic C. salmositica), 5 (nonpathogenic $C$. salmositica) and 9 ( $C$. bullocki) weeks p.i. Lactate levels were higher in pathogenic C. salmositica, than in nonpathogenic C. salmositica or C. bullocki 3-11 weeks p.i. and declined in all groups at 11 weeks (Table 3). In MEM-PR medium, lactate levels were higher in pathogenic $C$. salmositica in comparison to nonpathogenic $C$. salmositica or $C$. bullocki (Table 3).

\section{DISCUSSION}

After 11 weeks the phenol red in the culture of $C$. salmositica and C. bullocki had changed from red to yellow. An earlier study on Trypanosoma brucei bloodstream forms reported a similar colour change and this was attributed to the production of pyruvate, which lowered the $\mathrm{pH}$ of the medium and caused the colour change (Zinsstag et al. 1991). Pick and Keisari (1980) showed that macrophages released considerable amounts of hydrogen peroxide in response to phagocytic and other membrane stimuli. They noted that the phenol red in the medium changed from red to yellow whenever large amounts of hydrogen peroxide were present and that the change in colour was not due to a decrease in $\mathrm{pH}$.

Our results mirror those of Pick and Keisari (1980) however, there are some differences. Cryptobia spp. excrete pyruvate as a result of glucose metabolism but they also produce lactate. Cryptobia spp. have lactate dehydrogenase and thus are capable of converting pyruvate into lactate (Ardelli et al. 2000). Cryptobia (present study) and T. brucei (see Zinsstag et al. 1991) differ considerably in the amounts of pyruvate produced. If pyruvate decreased the $\mathrm{pH}$ in trypanosome culture then the low levels of pyruvate excreted by Cryptobia spp. cultures would explain the constant $\mathrm{pH}$ but not the colour changes noted in the present study.

We suggest that the colour change in the medium is due to production of hydrogen peroxide by Cryptobia spp. C. salmositica and C. bullocki excreted more hydrogen peroxide than either lactate or pyruvate. If either end-product of glycolysis were responsible for the colour change/acidity then these products were expected to increase with time. However lactate and pyruvate levels were low 11 weeks after culture while hydrogen peroxide levels were highest. The increased hydrogen peroxide in the MEM+PR medium paralleled the colour change. Many enzymatic reactions result in the generation of hydrogen peroxide as a by-product of the conversion of various substrates. The hydrogen peroxide produced is highly toxic and detoxification is mediated by catalase. In Cryptobia salmositica, catalase 
Table 1. In vitro production of hydrogen peroxide by pathogenic and nonpathogenic strains of Cryptobia salmositica and C. bullocki in medium with (MEM+PR) or without (MEM-PR) phenol red.

\begin{tabular}{|c|c|c|c|c|c|c|c|}
\hline \multirow{2}{*}{ Week } & \multicolumn{2}{|c|}{ Pathogenic C. salmositica } & \multicolumn{2}{c|}{ Nonpathogenic C. salmositica } & \multicolumn{2}{c|}{ C. bullocki } & \multirow{2}{*}{ P-value } \\
& MEM+PR & MEM-PR & MEM+PR & MEM-PR & MEM+PR & MEM-PR & \\
\hline 1 & $0.11 \pm 0.0004^{1}$ & $------{ }^{2}$ & $0.23 \pm 0.02$ & ------ & $0.27 \pm 0.05$ & ------ & 0.4878 \\
3 & $0.12 \pm 0.002$ & ------ & $4.27 \pm 0.31$ & ------ & $4.49 \pm 0.32$ & ------ & 0.4332 \\
5 & $0.65 \pm 0.03$ & ------ & $4.99 \pm 1.77$ & ----- & $5.36 \pm 1.34$ & ------ & 0.0733 \\
7 & $1.73 \pm 0.03$ & ------ & $5.36 \pm 1.49$ & ------ & $5.54 \pm 2.14$ & ------ & 0.0918 \\
9 & $9.73 \pm 1.54 *$ & ------ & $20.2 \pm 5.13$ & ----- & $19.6 \pm 4.27$ & ------ & 0.0385 \\
11 & $31.3 \pm 5.8$ & ------ & $64.0 \pm 9.3$ & ------ & $39.8 \pm 9.23$ & ------ & 0.0573 \\
\hline
\end{tabular}

${ }^{1}$ Mean \pm standard deviation of ten replicates expressed as $\mathrm{nM}$ of hydrogen peroxide produced per cell.

${ }^{2}$ Not detectable using the horseradish peroxidase assay.

${ }^{3} \mathrm{P}$-value among the groups (at each sampling period) evaluated at $\mathrm{p} \geq 0.05$.

* Significant difference.

Table 2. In vitro production of pyruvate by pathogenic and nonpathogenic strains of Cryptobia salmositica and C. bullocki in medium with (MEM+PR) or without (MEM-PR) phenol red.

\begin{tabular}{|c|c|c|c|c|c|c|c|}
\hline \multirow{2}{*}{ Week } & \multicolumn{2}{|c|}{ Pathogenic $C$. salmositica } & \multicolumn{2}{|c|}{ Nonpathogenic C. salmositica } & \multicolumn{2}{|c|}{ C. bullocki } & \multirow{2}{*}{ P-value ${ }^{2}$} \\
& MEM+PR & MEM-PR & MEM+PR & MEM-PR & MEM+PR & MEM-PR & \\
\hline 1 & $0.05 \pm 0.02^{1}$ & $0.07 \pm 0.03$ & $0.02 \pm 0.003$ & $0.06 \pm 0.01$ & $0.02 \pm 0.01$ & $0.03 \pm 0.01$ & 0.5803 \\
3 & $0.31 \pm 0.041$ & $0.6 \pm 0.06$ & $0.11 \pm 0.02$ & $0.4 \pm 0.09$ & $0.1 \pm 0.03$ & $0.91 \pm 0.04$ & 0.1057 \\
5 & $0.16 \pm 0.04$ & $0.15 \pm 0.09$ & $0.13 \pm 0.02$ & $0.7 \pm 0.07$ & $0.82 \pm 0.17$ & $0.58 \pm 0.08$ & 0.4082 \\
7 & $0.15 \pm 0.07^{*}$ & $0.93 \pm 0.09$ & $0.83 \pm 0.35$ & $0.68 \pm 0.04$ & $0.89 \pm 0.15$ & $0.72 \pm 0.02$ & 0.0251 \\
9 & $0.35 \pm 0.1$ & $0.13 \pm 0.02$ & $0.83 \pm 0.23^{*}$ & $0.13 \pm 0.05$ & $0.15 \pm 0.08$ & $0.11 \pm 0.04$ & 0.0497 \\
11 & $0.32 \pm 0.02$ & $0.1 \pm 0.06$ & $0.88 \pm 0.17^{*}$ & $1.75 \pm 0.42$ & $0.11 \pm 0.03$ & $0.10 \pm 0.05$ & 0.0308 \\
\hline
\end{tabular}

${ }^{1}$ Mean \pm standard deviation of ten replicates expressed as $\mathrm{nM}$ of pyruvate produced per cell.

${ }^{2} \mathrm{P}$-value among the groups (at each sampling period) evaluated at $\mathrm{p} \geq 0.05$.

* Significant difference.

Table 3. In vitro production of lactate by pathogenic and nonpathogenic strains of Cryptobia salmositica and C. bullocki in medium with (MEM+PR) or without (MEM-PR) phenol red.

\begin{tabular}{|c|c|c|c|c|c|c|c|}
\hline \multirow{2}{*}{ Week } & \multicolumn{2}{|c|}{ Pathogenic C. salmositica } & \multicolumn{2}{|c|}{ Nonpathogenic C. salmositica } & \multicolumn{2}{c|}{ C. bullocki } & \multirow{2}{*}{ P-value ${ }^{2}$} \\
& MEM+PR & MEM-PR & MEM+PR & MEM-PR & MEM+PR & MEM-PR & \\
\hline 1 & $5.9 \pm 1.7^{1}$ & $6.3 \pm 2.46$ & $10.5 \pm 6.02$ & $6.02 \pm 1.41$ & $8.3 \pm 2.7$ & $5.68 \pm 1.33$ & 0.4448 \\
3 & $36.9 \pm 4.28$ & $16.0 \pm 17.24$ & $13.3 \pm 2.31$ & $15.6 \pm 4.97$ & $12.2 \pm 3.9$ & $10.6 \pm 2.31$ & 0.3921 \\
5 & $16.7 \pm 2.58$ & $18.8 \pm 8.16$ & $15.4 \pm 3.57$ & $12.0 \pm 3.41$ & $13.6 \pm 4.8$ & $15.36 \pm 4.24$ & 0.2206 \\
7 & $14.8 \pm 3.49$ & $26.1 \pm 5.32$ & $10.3 \pm 1.75$ & $17.55 \pm 6.99$ & $10.6 \pm 3.4$ & $17.2 \pm 5.75$ & 0.0735 \\
9 & $34.8 \pm 6.82^{*}$ & $21.9 \pm 4.95$ & $14.5 \pm 1.93$ & $11.0 \pm 2.18$ & $16.2 \pm 4.6$ & $18.53 \pm 7.16$ & 0.0233 \\
11 & $4.3 \pm 0.16$ & $3.11 \pm 1.82$ & $3.71 \pm 0.056$ & $1.75 \pm 0.42$ & $1.55 \pm 1.4$ & $1.15 \pm 0.069$ & 0.0643 \\
\hline
\end{tabular}

\footnotetext{
${ }^{1}$ Mean \pm standard deviation of ten replicates expressed as $\mathrm{nM}$ of lactate produced per cell.

${ }^{2} \mathrm{P}$-value among the groups (at each sampling period) evaluated at $\mathrm{p} \geq 0.05$.

* Significant difference.
}

is mostly localised in glycosomes, but may be expelled into the cytoplasm of the cell (Ardelli et al. 2000). Cryptobia spp. can modify the catalase content in glycosomes in response to its environment (either in culture or in the blood of their host). Ardelli et al. (2000) suggested that the increase in catalase content was a response to accumulation of oxidative metabolites in culture, and that they included hydrogen peroxide. This hypothesis is supported in the present study.
Organic amine-based buffers such as Hepes are commonly used to increase the $\mathrm{H}^{+}$buffering power in a solution (Ganitkevich 1999) and $20 \mathrm{mM}$ Hepes buffer "clamps" the pH at 7.3 (Shigematsu and Arita 1999). Ardelli and Woo (1998) suggested that increasing the buffer capacity from $25 \mathrm{mM}$ to $100 \mathrm{mM}$ would extend the multiplication phase of Cryptobia spp. because the $\mathrm{pH}$ remained constant for a longer time. Our present data suggest that glucose and L-glutamine might be the 
limiting factors because the decline in parasite numbers in "old" cultures was not related to a change to an acidic environment. However, they were also sensitive to changes in $\mathrm{pH}$ and did not survive well in media without Hepes buffer (i.e. saline). Thus increasing the concentration of Hepes in the previous study increased available $\mathrm{H}^{+}$and might have stabilised the $\mathrm{pH}$, thus favouring enhanced parasite multiplication.

Acknowledgement. This study was supported by a grant from the National Science and Engineering Research Council of Canada (NSERC) to PTKW.

\section{REFERENCES}

ARCHER R.K. 1965: Haematological Techniques for Use on Animals. Blackwell Scientific Publications, Oxford, 159 pp.

ARDELLI B.F., WITT J.D.S., WOO P.T.K. 2000: Identification of glycosomes and metabolic end products in pathogenic and nonpathogenic strains of Cryptobia salmositica (Kinetoplastida: Bodonidae). Dis. Aquat. Org. 42: 41-51.

ARDELLI B.F., WOO P.T.K. 1998: Improved culture media for piscine haemoflagellates, Cryptobia and Trypanosoma (Kinetoplastida). J. Parasitol. 84: 1267-1271.

GANITKEVICH V.Y. 1999: Clearance of large $\mathrm{Ca}^{2+}$ loads in a single smooth muscle cell: examination of the role of mitochondrial $\mathrm{Ca}^{2+}$ uptake and intracellular $\mathrm{pH}$. Cell Calcium 25: 29-42.

LI S., WOO P.T.K. 1995: Efficacy of a live Cryptobia salmositica vaccine, and the mechanism of protection in vaccinated rainbow trout, Oncorhynchus mykiss, against cryptobiosis. Vet. Immunol. Immunopathol. 48: 343-353.

MARBACH E.P., WEIL M.H. 1967: Rapid enzymatic measurement of blood lactate and pyruvate. Use and significance of metaphosphoric acid as a common precipitant. Clin. Chem. 13: 314-325.

OPPERDOES F.R. 1995: Carbohydrate and energy metabolism in aerobic protozoa. In: J.J. Marr and M. Müller
(Eds.), Biochemistry and Molecular Biology of Parasites. Academic Press, London, pp. 19-32.

PICK E., KEISARI Y. 1980: A simple colorimetric method for the measurement of hydrogen peroxide produced by cells in culture. J. Immunol. Meth. 38: 161-170.

SHIGEMATSU S., ARITA M. 1999: Anoxia depresses sodium-calcium exchange currents in guinea-pig ventricular monocytes. J. Mol. Cell. Cardiol. 31: 895-906.

WOO P.T.K. 1979: Trypanoplasma salmositica: experimental infections in rainbow trout Salmo gairdneri. Exp. Parasitol. 47: 36-48.

WOO P.T.K., LI S. 1990: In vitro attenuation of Cryptobia salmositica and its use as a live vaccine against cryptobiosis in Oncorhynchus mykiss. J. Parasitol. 76: 752-755.

WOO P.T.K., POYNTON S.L. 1995: Diplomonadida, Kinetoplastida, and Amoebida (Phylum Sarcomastigophora). In: P.T.K. Woo (Ed.), Fish Diseases and Disorders I. Protozoan and Metazoan Infections. First Edition. CAB International, Oxford, pp. 27-96.

WOO P.T.K., THOMAS P.T. 1991: Polypeptide and antigen profiles of Cryptobia salmositica, C. bullocki and $C$. catostomi (Kinetoplastida: Sarcomastigophora) isolated from fishes. Dis. Aquat. Org. 11: 201-205.

ZINSSTAG R., BRUN R., GESSLER M. 1991: A new photometric assay for testing trypanocidal activity in vitro. Parasitol. Res. 77: 33-38.

Accepted 3 January 2001 